\title{
Detection of prions in the faeces of sheep naturally infected with classical scrapie
}

\author{
Linda A Terry ${ }^{1 *}$, Laurence Howells ${ }^{1}$, Keith Bishop² ${ }^{2}$ Claire A Baker ${ }^{3}$, Sally Everest ${ }^{1}$, Leigh Thorne ${ }^{1}$, Ben C Maddison ${ }^{3}$ \\ and Kevin C Gough ${ }^{4}$
}

\begin{abstract}
Classical scrapie is a naturally transmitted prion disease of sheep and goats. Contaminated environments may contribute to the spread of disease and evidence from animal models has implicated urine, blood, saliva, placenta and faeces as possible sources of the infection. Here we sought to determine whether sheep naturally infected with classical scrapie shed prions in their faeces. We used serial protein misfolding cyclic amplification (sPMCA) along with two extraction methods to examine faeces from sheep during both the clinical and preclinical phases of the disease and showed amplification of PrPSc in 7 of 15 and 14 of 14 sheep respectively. However PrPS was not amplified from the faeces of 25 sheep not exposed to scrapie. These data represent the first demonstration of prion shedding in faeces from a naturally infected host and thus a likely source of prion contamination in the environment.
\end{abstract}

\section{Introduction}

Prion diseases, or transmissible spongiform encephalopathies (TSEs), are fatal, neurological disorders that affect a range of mammalian species. They include scrapie in sheep, chronic wasting disease (CWD) in deer, bovine spongiform encephalopathy (BSE) in cattle and Creutzfeldt-Jakob disease (CJD) in humans. The "protein-only" hypothesis is the widely accepted paradigm for disease propagation and is based on the conversion of an apparently benign host prion protein $\left(\operatorname{PrP}^{\mathrm{C}}\right)$ into a pathological isoform, $\operatorname{PrP}^{\mathrm{Sc}}$, which constitutes the infectious agent [1]. To date $\operatorname{PrP}^{\mathrm{Sc}}$ is the only validated biochemical marker for prion diseases. These disorders are characterised by prolonged incubation periods before the onset of clinical signs, and the accumulation of $\mathrm{PrP}^{\mathrm{Sc}}$ particularly within the CNS. The sites of $\operatorname{PrP}^{\mathrm{Sc}}$ accumulation vary depending on the combination of host and prion strain under investigation, for instance classical ovine scrapie, cervid CWD and human vCJD display widespread distribution of $\mathrm{PrP}^{\mathrm{Sc}}$ within the CNS and lymphoreticular tissues (LRS) [2-4] whereas for cattle BSE, atypical scrapie and human CJD the accumulation of $\mathrm{PrP}^{\mathrm{Sc}}$ within LRS is much more restricted $[5,6]$.

\footnotetext{
* Correspondence: linda.terry@ahvla.gsi.gov.uk

${ }^{1}$ Animal Health and Veterinary Laboratories Agency, Woodham Lane, New Haw, Addlestone, Surrey, KT15 3NB, UK

Full list of author information is available at the end of the article
}

To date, studies investigating the shedding of the prion agent have focussed on scrapie and CWD. There is accumulating evidence for the excretion of prions within milk $[7,8]$, urine $[9,10]$, saliva [10-12] and faeces [13]. Such reports utilise methodologies that are exquisitely sensitive in their detection of prion infectivity or $\mathrm{PrP}^{\mathrm{Sc}}$, namely bioassay or amplification of $\operatorname{PrP}^{\mathrm{Sc}}$ by serial protein misfolding cyclic amplification (sPMCA). PMCA was pioneered by Soto et al. [14] in a rodent model of scrapie and this is an in vitro technique that amplifies trace amounts of $\operatorname{PrP}^{\mathrm{Sc}}$ within a test sample during iterative rounds of sonication and incubation at $37^{\circ} \mathrm{C}$. Importantly, this technique has been adapted for the high level amplification of $\operatorname{PrP}^{\mathrm{Sc}}$ from natural sources of prion infection including ovine scrapie [15], bovine BSE [16], human CJD [17] and cervid CWD [18].

There is evidence from experimentally infected animals that prions are excreted in faeces. Safar et al. [13] used a rodent scrapie model to demonstrate the presence of prions in faeces when measured by conformation-dependent immunoassay (CDI) and transgenic mouse bioassay. Prions were present in faeces from hamsters throughout disease incubation. An analogous study in hamsters employed the sPMCA amplification of prions in faecal extracts and demonstrated the presence of prions during clinical disease [19]. In addition, experimentally infected mule deer 
excreted prion infectivity in faeces during preclinical and clinical stages of CWD [20].

Here, we apply sPMCA to investigate the excretion of prions within the faeces of naturally infected sheep, both in the preclinical and clinical stages of disease progression.

\section{Materials and methods Samples}

Faecal samples were removed directly from the gut at post mortem or from the rectum of live animals while avoiding contamination with blood. Visible contamination with blood was not observed in any of the samples. This method of collection also minimised any contamination from the environment. Samples were stored at $-20^{\circ} \mathrm{C}$ until assayed. Faeces were collected from sheep homozygous for VRQ (codons 136, 154 and 171 respectively of the PRNP gene) at 9 to 10 months of age and at clinical end-point (mean survival time of 22 months of age). As negative controls, faeces were collected from VRQ/VRQ unexposed sheep from a VLA New Zealand derived flock. No consistent differences in the consistency of the faeces were observed between exposed and negative control sheep.

\section{Faeces suspension preparation}

Method 1 [Silicon dioxide $\left(\mathrm{SiO}_{2}\right)$ ]: After thawing, faecal pellets were transferred to $50 \mathrm{~mL}$ disposable tubes for homogenisation and diluted to three times the original mass using phosphate buffered saline (PBS, $15 \mathrm{mM}$ $\mathrm{KH}_{2} \mathrm{PO}_{4}, 81 \mathrm{mM} \mathrm{Na} 2 \mathrm{HPO}_{4}, 137 \mathrm{mM} \mathrm{NaCl}$, and $3 \mathrm{mM}$ $\mathrm{KCl}$ ) containing $2 \times$ miniprotease inhibitors (without EDTA, Roche, Welwyn Garden City, UK). Each faecal suspension was vortexed for 5 to 10 min until homogeneous and $0.5 \mathrm{~mL}$ aliquots of this $33 \%$ faecal homogenate were transferred to a $15 \mathrm{~mL}$ falcon tube and stored at $-80^{\circ} \mathrm{C}$ until required.

Method 2 [Sodium Phosphotungstic acid (NaPTa)]: After thawing the faecal samples were transferred to disposable tubes for homogenisation and diluted 1:9 in water containing protease inhibitors (Roche). The samples were then homogenised using the Prionics PrioGENISER $^{\text {TM }}$ (Prionics, Zurich, Switzerland) twice for $40 \mathrm{~s}$ at maximum speed. Prions were then extracted from faecal homogenates using NaPTa.

\section{Extraction of prions}

Method $1\left[\mathrm{SiO}_{2}\right]$ : Faecal homogenates $(0.5 \mathrm{~mL}$ of a $33 \%$ $\mathrm{w} / \mathrm{v}$ faecal homogenate) were diluted $1: 1$ in $2 \times$ phosphate buffer ( $\mathrm{pH}$ 7) containing 1\% deoxycholate (DOC) and $1 \% \mathrm{NP} 40$, then further diluted to $5 \mathrm{~mL}$ with $1 \times \mathrm{PBS}$ containing $0.5 \%$ DOC and $0.5 \%$ NP40. The faecal homogenates were then incubated for $1 \mathrm{~h}$ at room temperature (RT). Following centrifugation at $1500 \mathrm{~g}$ for $3 \mathrm{~min}$ the supernatant was removed and the pellet discarded.
$80 \mu \mathrm{L}$ of a $20 \%(\mathrm{w} / \mathrm{v}) \mathrm{SiO}_{2}$ suspension were added to the $5 \mathrm{~mL}$ faecal supernatant and the sample rotated gently for $2 \mathrm{~h}$ at RT. The samples were centrifuged at $700 \mathrm{~g}$ for $4 \mathrm{~min}$ and the supernatant discarded. The $\mathrm{SiO}_{2}$ pellets were washed by gentle pipetting using $1 \mathrm{~mL}$ $0.1 \%$ SDS.

Method 2 [NaPTa]: Faecal homogenates $(0.5 \mathrm{~mL}$ of a $10 \% \mathrm{w} / \mathrm{v}$ homogenate in deionised water containing protease inhibitors (mini-pill complete, Roche)) were transferred to homogenisation tubes and sodium dodecyl sulphate (SDS) added to a final concentration of $1 \%$. The samples were then ribolysed (BioRad, Hemel Hempstead, UK) three times for $45 \mathrm{~s}$ each and cooled between each cycle. The samples were incubated at RT for $1 \mathrm{~h}$ rotating gently. Homogenates were then centrifuged for $20 \mathrm{~min}$ at $4^{\circ} \mathrm{C}$ at $15000 \mathrm{~g}$ and the supernatants transferred to a clean tube. The pellets were discarded. The supernatant was diluted 1:1 with PBS containing 4\% N-Lauroylsarcosine sodium salt (Sigma, Poole, UK) and treated with Benzonaze ( 1 unit $/ \mu \mathrm{L}$, Sigma) for $30 \mathrm{~min}$ at $50^{\circ} \mathrm{C}$. Following treatment, $\mathrm{NaPTa}$ was added to a final concentration of $0.57 \%$ and the sample incubated at $37^{\circ} \mathrm{C}$ rotating gently for $30 \mathrm{~min}$. Samples were centrifuged at $15000 \mathrm{~g}$ for 15 min at $10^{\circ} \mathrm{C}$ and the supernatant discarded. Pellets were frozen at $-80^{\circ} \mathrm{C}$. Upon analyses, pellets were resuspended in $50 \mu \mathrm{L}$ of PMCA buffer (PBS, additional $150 \mathrm{mM} \mathrm{NaCl}$, 4 mM EDTA, pH8.0, 1.0\% (v/v) Triton X-100 and miniprotease inhibitor, Roche).

\section{Amplification of prions}

Method $1\left[\mathrm{SiO}_{2}\right]$ : Faecal extracts derived from $\mathrm{SiO}_{2}$ precipitation were centrifuged at $16000 \mathrm{~g}$ for $3 \mathrm{~min} .10 \mu \mathrm{L}$ of each faecal extract supernatant (from the equivalent of $8.3 \mathrm{mg}$ wet weight of faeces) were mixed 1:9 with normal ovine brain homogenate. The samples were then subjected to $40 \mathrm{~s}$ cycles of sonication every $30 \mathrm{~min}$ incubating at $37^{\circ} \mathrm{C}$ for $24 \mathrm{~h}$ (1 round), after which the amplified samples were diluted 1:2 with PMCA substrate in a final volume of $100 \mu \mathrm{L}$ and the sample subjected to further rounds of PMCA. Ten rounds of amplification were carried out. The amplification product was stored at $-20^{\circ} \mathrm{C}$. Samples from sheep incubating scrapie as well as from sheep not exposed to the scrapie agent were analyzed concurrently within the same run on the same sonicator. Each $\mathrm{SiO}_{2}$ extract was amplified within two separate experiments. After 10 rounds of amplification in the absence of PolyA no de novo synthesis was observed. Amplification was not extended beyond 10 rounds.

Method 2 [NaPTA]: Faecal extracts derived from NaPTa precipitation ( $8 \mu \mathrm{L}$; from the equivalent of $8 \mathrm{mg}$ wet weight of faeces) were mixed 1:9 with normal ovine brain homogenate (obtained at post mortem from a VRQ/VRQ sheep from the New Zealand-derived VLA 
scrapie-free flock; a $10 \%(\mathrm{w} / \mathrm{v})$ homogenate in PMCA buffer) supplemented with synthetic polyA RNA to a final concentration $100 \mu \mathrm{g} / \mathrm{mL}$. The samples were then subjected to $40 \mathrm{~s}$ cycles of sonication every $30 \mathrm{~min}$ incubating at $37^{\circ} \mathrm{C}$ for $24 \mathrm{~h}$ ( 1 round). The sonicated product was then replenished 1:4 with fresh normal brain homogenate and sonication repeated for a further $24 \mathrm{~h}$. This was repeated until four rounds were completed. Using this PMCA protocol rounds were restricted to 4 because de novo synthesis was observed at 6 rounds or more under $\operatorname{PrP}^{\mathrm{Sc}}$ free conditions (data not shown). No de novo synthesis was observed in the presence of PolyA at round 4 . The amplification products were stored after all PMCA rounds at $-20^{\circ} \mathrm{C}$ until tested for $\mathrm{PrP}^{\mathrm{Sc}}$ content.

\section{Analysis of $\mathrm{PrP}^{\mathrm{Sc}}$}

PMCA products were tested using the IDEXX HerdChek antigen test for scrapie as directed by the manufacturers. Positives were scored using the manufacturer's cut-off values. Alternatively, PMCA samples were digested with $50 \mu \mathrm{g} / \mathrm{mL}$ proteinase $\mathrm{K}, 0.045 \%(\mathrm{w} / \mathrm{v})$ SDS for $1 \mathrm{~h}$ at $37^{\circ} \mathrm{C}$ before analysis by Western blotting using $12 \%(\mathrm{w} / \mathrm{v})$ NuPAGE pre-cast Bis-Tris gels as previously described [21].

\section{Statistical analysis}

When comparing the percentage of positive sPMCA reactions for different cohorts of samples, data were set up as $2 \times 2$ contingency tables and Fisher's exact test (one-tailed) was applied to derive $p$-values.

\section{Results}

Faeces were sampled directly from the transverse colon of clinically-affected sheep at post mortem or from the rectum of live sheep with preclinical scrapie. Prions were extracted from faeces employing a mild detergent extraction $\left(0.5 \% \mathrm{w} / \mathrm{v}\right.$ DOC and NP40) followed by $\mathrm{SiO}_{2}$ precipitation. Prions extracted using $\mathrm{SiO}_{2}$ were amplified over 10 rounds of sPMCA before attempted detection of $\operatorname{PrP}^{\mathrm{Sc}}$ by Western blot. This method was previously shown to amplify prions from the saliva of sheep incubating scrapie [11]. Here, when applied to the faeces from scrapie infected animals, $\mathrm{PrP}^{\mathrm{Sc}}$ was amplified from 6 of 29 animals: four animals with clinical disease and two animals which were clinically normal at approximately the midpoint of disease incubation (10 positive sPMCA reactions from 58 analyses; Figure 1, Table 1). No prions were amplified from faeces collected from sheep which had not been exposed to the scrapie agent (10 animals, 60 analyses, Figure 1, Table 1). These data demonstrate that prions were secreted in faeces from sheep incubating natural scrapie $(p=0.001)$.
In order to estimate the levels of scrapie prions present within faeces, the limit of detection of the methodology was determined for the earliest sPMCA round that amplified $\mathrm{PrP}^{\mathrm{Sc}}$ from both preclinical and clinical faecal samples. Using $\mathrm{SiO}_{2}$ extraction coupled with sPMCA amplification of prions in the absence of polyA RNA, seven rounds of sPMCA amplification yielded detectable $\mathrm{PrP}^{\mathrm{Sc}}$ for 4 of the samples, 3 taken from clinical animals $(0320 / 09,0324 / 09,0572 / 09)$ and one taken from an ani$\mathrm{mal}$ in the preclinical stage of disease (1305/09). A dilution series of a brain homogenate from an animal with terminal scrapie was spiked into a faecal homogenate and then extracted and amplified. Extraction followed by amplification for 7 rounds of sPMCA detected $\operatorname{PrP}^{\mathrm{Sc}}$ down to $0.64 \mathrm{ng}$ of brain material spiked into $167 \mathrm{mg}$ wet weight of faeces (data not shown).

To investigate further the shedding of prions in faeces, a second method of extraction and amplification was developed. This method used potentially more stringent extraction conditions and enhanced sPMCA amplification. Briefly, prions were eluted from faeces with $1 \%$ SDS followed by NaPTa precipitation of prions and sPMCA amplification employing polyA;-the presence of polyA having been shown to increase the sensitivity of the sPMCA amplification [15,22]. The presence of $\mathrm{PrP}^{\mathrm{Sc}}$ was demonstrated in the amplification product using the IDEXX HerdChek antigen assay for scrapie (Table 1). $\mathrm{PrP}^{\mathrm{Sc}}$ was detected in the faeces of all 14 sheep which were in the preclinical stages of scrapie and sampled at 9 to 10 months of age (19 positive sPMCA reactions from 22 analyses; Table 1). Of the sheep at clinical end-stage, $\operatorname{PrP}^{\mathrm{Sc}}$ was amplified from the faeces of 6 out of 15 sheep (8 out of 27 analyses were positive). Analyses of animals not exposed to the scrapie agent again demonstrated that no prions could be detected ( 25 animals, 45 analyses; Table 1 and data not shown). These data indicate that scrapie prions are excreted in faeces in both preclinical $(p<0.001)$ and clinical $(p<0.001)$ stages of the disease. $\mathrm{PrP}^{\mathrm{Sc}}$ was detected at the earliest following round 4 using this method except in 1 sample (324/09) where $\operatorname{PrP}^{\mathrm{Sc}}$ was detected following round 3 .

These data report for the first time the excretion of prions in the faeces of naturally infected animals and further support the role of the faecal-oral route as a means of horizontal transmission in the field.

\section{Discussion}

The reported study demonstrates the presence of prions in the faeces of sheep naturally infected with scrapie. Additionally, $\operatorname{PrP}^{\mathrm{Sc}}$ was present in the faeces of sheep both in the terminal stages of disease and when in the early preclinical phase, suggesting that prions are likely to be shed into the environment throughout pathogenesis. These 


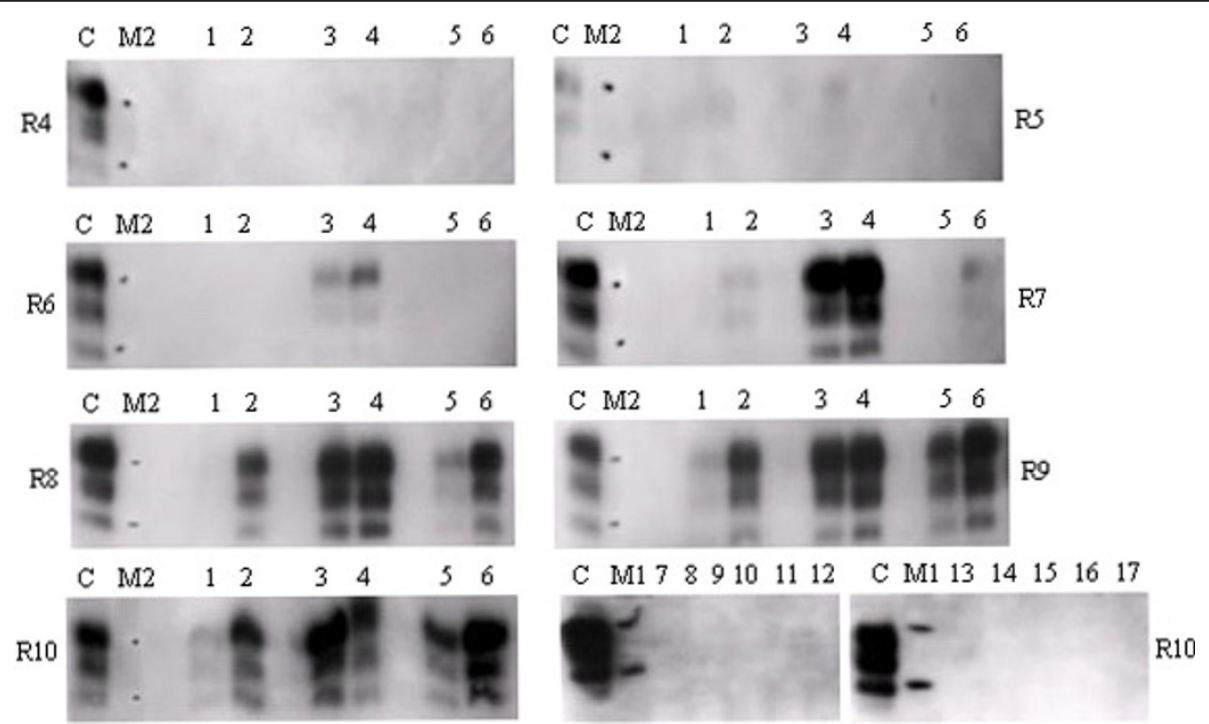

Figure 1 Prion excretion in faeces of healthy and clinical scrapie infected sheep. Prions extracted from 8.3 mg of faeces were used as seed for Method 1 [10 rounds of sPMCA]. After 4 to 10 rounds of sPMCA (as indicated), products were digested with proteinase $\mathrm{K}$ and $10 \mu \mathrm{L}$ applied to Western blots. PrP was detected with monoclonal antibodies SHA31 and P4; molecular weight markers are shown (M1, 20 and 30 kDa; M2, 19 and $28 \mathrm{kDa}$ ). Sheep were either in the preclinical (lanes 1 and 2 for animals 1236/09 and 1305/09 respectively) or clinical stages of disease progression (lanes 3-6 for animals 0320/09, 0324/09, 0470/09 and 0572/09 respectively) or were unexposed to the scrapie agent (lanes 7-16 for animals R185, R223, R233, R234, R297, R303, R328, R382, R413 and R421 respectively). Two analyses were carried out for each sample and representative analyses are shown. Cerebellum ( $2.5 \mathrm{\mu L}$ of a $10 \% \mathrm{w} / \mathrm{v}$ homogenate) from a terminally affected sheep was analysed on each blot as a PrP ${ }^{\mathrm{SC}}$ control (C).

data concur with a study of mule deer experimentally infected with CWD [20] where infectivity was observed in faecal samples collected from the deer from nine months after inoculation and maintained during the clinical phase several months later. Together these data are fully consistent with faeces being a source of environmental prion contamination that contributes to transmission for both scrapie and CWD.

Within the present study, two distinct methods were applied to detect faecal $\mathrm{PrP}^{\mathrm{Sc}}$. Prions were detected less frequently when employing mild detergent extraction, $\mathrm{SiO}_{2}$ precipitation and a standard sPMCA amplification, compared to when using SDS extraction, NaPTa precipitation and sPMCA incorporating polyA. This may indicate that the latter method has an improved sensitivity. The method based on mild extraction and nonenhanced sPMCA detected prions more frequently in clinical samples and in contrast the method based on SDS extraction and polyA-enhanced amplification detected prions more frequently in preclinical samples. This suggests that preclinical animals may be more likely to secrete prions compared to animals with clinical disease, but that the levels of prion present within faeces is higher in the clinical animals. With the mild extraction conditions amplification of prions was observed at earlier rounds in samples from clinical compared to preclinical animals, supporting the proposition that prions are at higher levels in faeces during clinical disease.

Several sources of prions in faeces could be postulated including environmental ingestion and swallowing of infected saliva [11]. However, the most likely source is shedding from the gut-associated lymphoid tissue. Early and rapid accumulation of $\operatorname{PrP}^{\mathrm{Sc}}$ in the Peyer's patches is consistent with the detection of prions in faeces during the preclinical phase. In sheep with a VRQ/VRQ PRNP genotype this accumulation commences at around 2-3 months of age. Ruminants have specialised Peyer's patches that are continuous throughout the length of the ileum amounting to approximately 100000 follicles and all of these could potentially be infected and shedding prions into the lumen. However the number of follicles diminishes at around 12-15 months of age thus reducing the potential for shedding at this site in older sheep such as those with clinical disease in the present study. Additionally, the recto-anal mucosa associated lymphoid tissue may also play a role in contributing to the levels of $\mathrm{PrP}^{\mathrm{Sc}}$ found in the faeces; in the present study this route would not contribute to prion in the samples from clinical sheep due to direct sampling from the gut. These factors would be expected to result in lower prion levels and/or a lower frequency of prion excretion within the clinical faecal samples. However, this is not supported by the presented data indicating that levels of prion 
Table 1 Amplification of PrP ${ }^{\mathrm{Sc}}$ from sheep faeces

\begin{tabular}{|c|c|c|c|}
\hline Origin of faecal sample & $\begin{array}{l}\text { Animal } \\
\text { ID }\end{array}$ & $\begin{array}{l}\text { number positive/total number of analyses } \\
\text { Method } 1^{\mathrm{a}}\end{array}$ & $\begin{array}{l}\text { number of positive/total number of analyses } \\
\text { Method } 2^{\mathrm{b}}\end{array}$ \\
\hline & R382 & $0 / 6$ & $0 / 2$ \\
\hline & R328 & $0 / 6$ & $0 / 2$ \\
\hline & R234 & $0 / 6$ & $0 / 2$ \\
\hline & R413 & $0 / 6$ & $0 / 2$ \\
\hline & R421 & $0 / 6$ & $0 / 2$ \\
\hline \multirow[t]{12}{*}{ Unexposed sheep } & R185 & $0 / 6$ & $0 / 1$ \\
\hline & R223 & $0 / 6$ & $0 / 1$ \\
\hline & R233 & $0 / 6$ & $0 / 1$ \\
\hline & R297 & $0 / 6$ & $0 / 1$ \\
\hline & R303 & $0 / 6$ & $0 / 1$ \\
\hline & Total & $0 / 60$ & $0 / 15^{c}$ \\
\hline & $1203 / 09$ & $0 / 2$ & $2 / 2(4,4)$ \\
\hline & $1236 / 09$ & $1 / 2(9)^{d}$ & $1 / 2(4)$ \\
\hline & $1252 / 09$ & $0 / 2$ & $2 / 2(4,4)$ \\
\hline & $1271 / 09$ & $0 / 2$ & $2 / 2(4,4)$ \\
\hline & $1284 / 09$ & $0 / 2$ & $2 / 2(4,4)$ \\
\hline & $1292 / 09$ & $0 / 2$ & $2 / 2(4,4)$ \\
\hline \multirow{16}{*}{$\begin{array}{l}\text { Preclinical exposed sheep age 9-10 } \\
\text { months }\end{array}$} & $1298 / 09$ & $0 / 2$ & $1 / 2(4)$ \\
\hline & $1305 / 09$ & $2 / 2(7,10)$ & $1 / 2(4)$ \\
\hline & $1227 / 09$ & $0 / 2$ & $1 / 1(4)$ \\
\hline & $1279 / 09$ & $0 / 2$ & $1 / 1(4)$ \\
\hline & $1286 / 09$ & $0 / 2$ & $1 / 1(4)$ \\
\hline & $1296 / 09$ & $0 / 2$ & $1 / 1(4)$ \\
\hline & $1299 / 09$ & $0 / 2$ & $1 / 1(4)$ \\
\hline & $1307 / 09$ & $0 / 2$ & $1 / 1(4)$ \\
\hline & Total & $3 / 28$ & $19 / 22$ \\
\hline & $317 / 09$ & $0 / 2$ & $0 / 2$ \\
\hline & $319 / 09$ & $0 / 2$ & $1 / 2(4)$ \\
\hline & $320 / 09$ & $2 / 2(6,6)$ & $1 / 2(4)$ \\
\hline & $324 / 09$ & $1 / 2(6)$ & $2 / 2(3,4)$ \\
\hline & $421 / 09$ & $0 / 2$ & $0 / 2$ \\
\hline & $352 / 09$ & $0 / 2$ & $0 / 2$ \\
\hline & $353 / 09$ & $0 / 2$ & $0 / 2$ \\
\hline \multirow[t]{9}{*}{ Sheep with clinical scrapie } & $354 / 09$ & $0 / 2$ & $0 / 2$ \\
\hline & $359 / 09$ & $0 / 2$ & $1 / 2(4)$ \\
\hline & $382 / 09$ & $0 / 2$ & $0 / 2$ \\
\hline & $457 / 09$ & $0 / 2$ & $0 / 2$ \\
\hline & $470 / 09$ & $2 / 2(8,9)$ & $2 / 2(4,4)$ \\
\hline & $606 / 09$ & $0 / 2$ & $0 / 1$ \\
\hline & $608 / 09$ & $0 / 2$ & $1 / 1(4)$ \\
\hline & $572 / 09$ & $2 / 2(7,9)$ & $0 / 1$ \\
\hline & Total & $7 / 30$ & $8 / 27$ \\
\hline
\end{tabular}

${ }^{a}$ Method 1: Prion was extracted in NP40 and DOC, precipitated with $\mathrm{SiO}_{2}$ and amplified by sPMCA for 10 rounds.

${ }^{b}$ Method 2: Prions were extracted in SDS, NaPTa precipitated and amplified by sPMCA in the presence of polyA for 4 rounds.

${ }^{\mathrm{C}}$ Faeces from a further 15 animals was analysed in duplicate by this method and no prions were detected (data not shown).

${ }^{\mathrm{d}}$ The sPMCA round at which the sample first became positive is indicated in parenthesis. 
excretion is not solely influenced by excretion from RAMALT or the ileal Peyer's patches.

Not all prion diseases result in infection of the gutassociated lymphoid tissue. The low levels of prion infection in the gut of cattle with natural BSE [23] could explain the apparent, fortuitous failure of lateral spread of BSE within herds.

Here, with the estimated levels of prion shed within the faeces combined with an estimated ovine faecal output of $67 \mathrm{~kg} /$ month [24] it could be speculated that between 9 months and 22 months of age sheep could excrete prions equivalent to the potentially infectious load within approximately $3.3 \mathrm{mg}$ of clinically infected brain. Tamguney et al. [20] used bioassay to estimate the levels of infectivity in faeces from experimentally-infected CWD deer; over a 10 month period these levels were estimated to approach that present within a clinically infected whole brain. These levels are much higher than those found in the present study with scrapie infected sheep; however, this could reflect differences between the prion strains and host species under investigation or be due to differences in the prion detection methodologies employed.

Previous studies have indicated that risk factors for horizontal transmission in the field involve contact during the postnatal period [25-27]. However, exposure to pasture that has been grazed by infected sheep is sufficient to transmit disease even in the absence of lambing $[25,28]$. The findings of the present study would suggest that prions in faeces will contribute to this contamination and support the role of the faecal-oral route as a means of horizontal transmission. Strategies for the control of scrapie will need to take this into account particularly for goats where successful elimination is still reliant on depopulation, disinfection and restocking.

In North America, the presence of prions in secreta and excreta of deer and the resultant contamination of the environment is likely to have contributed to the spread of CWD and this is likely to progress unabated given the estimation of the amount of prions contained within cervine faeces [20]. Therefore environmental contamination of pasture remains a serious impediment to future eradication of prion disease in both Europe and in North America.

\footnotetext{
Acknowledgements

We thank the TSE Archive at the VLA (Addlestone, Surrey, UK) for the provision of ruminant brain material, all the staff in the animal services unit and pathology for their contribution in the collection of samples and maintenance of the flock and to Susan Bellworthy for her input at the initiation of this project. This work was funded by the Department for Environment, Food and Rural Affairs, UK.
}

\section{Author details}

'Animal Health and Veterinary Laboratories Agency, Woodham Lane, New Haw, Addlestone, Surrey, KT15 3NB, UK. ${ }^{2}$ ADAS UK, School of Veterinary Medicine and Science, The University of Nottingham, Sutton Bonington
Campus, College Road, Sutton Bonington, Leicestershire, LE12 5RD, UK. ${ }^{3}$ ADAS UK, Department of Biology, University of Leicester, University Road, Leicester, LE1 7RH, UK. ${ }^{4}$ School of Veterinary Medicine and Science, The University of Nottingham, Sutton Bonington Campus, College Road, Sutton Bonington, Leicestershire, LE12 5RD, UK.

\section{Authors' contributions}

LT contribute to the design and coordination of the study and prepared the manuscript. LH carried out all experimental work for method 2. KB carried out all experimental work for method 1. CB carried out method development work for method 1. SE contributed to the coordination of the study and analyses of results from method 2. LTh initiated and designed the PMCA studies. BM contributed to the coordination of the study and analyses of results from method 1. KG contributed to the coordination of the study and analyses of results from method 1 and assisted in manuscript preparation. All authors read and approved the final manuscript.

\section{Competing interests}

The authors declare that they have no competing interests.

Received: 22 December 2010 Accepted: 18 May 2011

Published: 18 May 2011

\section{References}

1. Prusiner SB: Prions. Proc Natl Acad Sci USA 1998, 95(23):13363-13383.

2. Langeveld JP, Jacobs JG, Erkens JH, Bossers A, van Zijderveld FG, van Keulen L: Rapid and discriminatory diagnosis of scrapie and BSE in retro-pharyngeal lymph nodes of sheep. BMC Vet Res 2006, 2:19.

3. Wadsworth JDF, Joiner S, Hill AF, Campbell TA, Desbruslais M, Luthert PJ, Collinge J: Tissue distribution of protease resistant prion protein in variant Creutzfeldt-Jakob disease using a highly sensitive immunoblotting assay. Lancet 2001, 358(9277):171-180.

4. Fox KA, Jewell JE, Williams ES, Miller MW: Patterns of PrPCWD accumulation during the course of chronic wasting disease infection in orally inoculated mule deer (Odocoileus hemionus). J Gen Virol 2006, 87(Pt 11):3451-3461.

5. Wells GAH, Hawkins SAC, Green RB, Austin AR, Dexter I, Spencer $Y$ I, Chaplin MJ, Stack MJ, Dawson M: Preliminary observations on the pathogenesis of experimental BSE: an update. Vet Rec 1998, 142:103-106

6. Hill AF, Butterworth RJ, Joiner S, Jackson G, Rossor MN, Thomas DJ, Frosh A, Tolley N, Bell JE, Spencer M, King A, Al-Sarraj S, Ironside JW, Lantos PL, Collinge J: Investigation of variant Creutzfeldt-Jakob disease and other human prion diseases with tonsil biopsy samples. Lancet 1999, 353(9148):183-189.

7. Konold T, Moore SJ, Bellworthy SJ, Simmons HA: Evidence of scrapie transmission via milk. BMC Vet Res 2008, 4(1):14.

8. Maddison BC, Baker CA, Rees HC, Terry LA, Thorne L, Bellworthy SJ, Whitelam GC, Gough KC: Prions are secreted in milk from clinically normal scrapie-exposed sheep. J Virol 2009, 83(16):8293-8296.

9. Gonzalez-Romero D, Barria MA, Leon P, Morales R, Soto C: Detection of infectious prions in urine. FEBS Lett 2008, 582(21-22):3161-3166.

10. Haley NJ, Seelig DM, Zabel MD, Telling GC, Hoover EA: Detection of CWD prions in urine and saliva of deer by transgenic mouse bioassay. PLOS One 2009, 4(3):e4848.

11. Maddison BC, Rees HC, Baker CA, Taema M, Bellworthy SJ, Thorne L, Terry LA, Gough KC: Prions are secreted into the oral cavity in sheep with preclinical scrapie. J Infect Dis 2010, , 11: 1672-1676.

12. Mathiason CK, Powers JG, Dahmes SJ, Osborn DA, Miller KV, Warren RJ, Mason GL, Hays SA, Hayes-Klug J, Seelig DM, Wild MA, Wolfe LL, Spraker TR, Miller MW, Sigurdson CJ, Telling GC, Hoover EA: Infectious prions in the saliva and blood of deer with chronic wasting disease. Science 2006, 314(5796):133-136.

13. Safar JG, Lessard P, Tamguney G, Freyman Y, Deering C, Letessier F, Dearmond SJ, Prusiner SB: Transmission and detection of prions in feces. $J$ Infect Dis 2008, 198(1):81-89.

14. Saborio GP, Permanne B, Soto C: Sensitive detection of pathological prion protein by cyclic amplification of protein misfolding. Nature 2001, 411(6839):810-813

15. Thorne L, Terry LA: In vitro amplification of PrPSc derived from the brain and blood of sheep infected with scrapie. J Gen Virol 2008, 89(Pt 12):3177-3184. 
16. Gough KC, Baker CA, Taema M, Maddison BC: In vitro amplification of prions from milk in the detection of subclinical infections. Prion 2009, 3(4):236-239.

17. Jones M, Peden AH, Prowse CV, Groner A, Manson JC, Turner ML, Ironside JW, MacGregor IR, Head MW: In vitro amplification and detection of variant Creutzfeldt-Jakob disease PrP ${ }^{\text {Sc }}$. J Pathol 2007, 213(1):21-26.

18. Kurt TD, Perrott MR, Wilusz CJ, Wilusz J, Supattapone S, Telling GC, Zabel MD, Hoover EA: Efficient in vitro amplification of chronic wasting disease PrPRES. J Virol 2007, 81(17):9605-9608

19. Kruger D, Thomzig A, Lenz G, Kampf K, McBride P, Beekes M: Faecal shedding, alimentary clearance and intestinal spread of prions in hamsters fed with scrapie. Vet Res 2009, 40(1):4

20. Tamguney G, Miller MW, Wolfe LL, Sirochman TM, Glidden DV, Palmer C, Lemus A, DeArmond SJ, Prusiner SB: Asymptomatic deer excrete infectious prions in faeces. Nature 2009, 461(7263):529-532.

21. Rees HC, Maddison BC, Owen JP, Whitelam GC, Gough KC: Concentration of disease-associated prion protein with silicon dioxide. Mol Biotechnol 2009, 41(3):254-262.

22. Deleault NR, Lucassen RW, Supattapone S: RNA molecules stimulate prion protein conversion. Nature 2003, 425(6959):717-720

23. Terry LA, Marsh S, Ryder SJ, Hawkins SA, Wells GA, Spencer YI: Detection of disease-specific PrP in the distal ileum of cattle exposed orally to the agent of bovine spongiform encephalopathy. Vet Rec 2003, 152(13):387-392.

24. Smith KA, Frost JP: Nitrogen excretion by farm livestock with respect to land spreading requirements and controlling nitrogen losses to ground and surface waters. Part 1: cattle and sheep. Biosresource Technol 2000, 71:173-181.

25. Dexter G, Tonque SC, Heasman L, Bellworthy SJ, Davis A, Moore SJ, Simmons MM, Sayers AR, Simmons HA, Matthews D: The evaluation of exposure risks for natural transmission of scrapie within an infected flock. BMC Vet Res 2009, 5:38.

26. Touzeau S, Chase-Topping ME, Matthews L, Lajous D, Eychenne F, Hunter N, Foster JD, Simm G, Elsen JM, Woolhouse ME: Modelling the spread of scrapie in a sheep flock: evidence for increased transmission during lambing seasons. Arch Virol 2006, 151(4):735-751.

27. Woolhouse ME, Stringer SM, Matthews L, Hunter N, Anderson RM: Epidemiology and control of scrapie within a sheep flock. Proc Biol Sci 1998, 265(1402):1205-1210

28. Ryder $S$, Dexter $G$, Bellworthy $S$, Tongue $S$ : Demonstration of lateral transmission of scrapie between sheep kept under natural conditions using lymphoid tissue biopsy. Res Vet Sci 2004, 76(3):211-217.

doi:10.1186/1297-9716-42-65

Cite this article as: Terry et al:: Detection of prions in the faeces of sheep naturally infected with classical scrapie. Veterinary Research 2011 42:65.

\section{Submit your next manuscript to BioMed Central and take full advantage of:}

- Convenient online submission

- Thorough peer review

- No space constraints or color figure charges

- Immediate publication on acceptance

- Inclusion in PubMed, CAS, Scopus and Google Scholar

- Research which is freely available for redistribution 